J. Lake Sci. (湖泊科学), 2013, 25(5):628-634

http: //www.jlakes.org. E-mail : jlakes@niglas.ac.cn

(C) 2013 by Journal of Lake Sciences

\title{
蓝藻对太湖底泥反硝化过程的影响和机理分析”
}

\author{
黎丽雯 ${ }^{1,2}$, 潘 纲 $^{2 * *}$, 李 梁 $^{2}$, 李 宏 $^{2}$, 施文卿 $^{2}$, 张洪刚 $^{2}$, 朱广伟 ${ }^{* * *}$ \\ ( 1 : 中国科学院南京地理与湖泊研究所, 南京 210008) \\ (2: 中国科学院生态环境研究中心, 北京 100085)
}

\begin{abstract}
摘 要: 通过模拟实验, 研究了因自然或人工沉降的太湖蓝藻在厌氧条件下作为碳源对底泥微生物反硝化脱氮的促进作 用. 通过对底泥总氮、化合态氮素、挥发性脂肪酸 (VFAs)、COD、电位和 $\mathrm{pH}$ 等指标的监测, 发现藻体中大量的生物可降解 碳素在厌氧消解后产生挥发性脂肪酸等一些可供反硝化菌直接利用的小分子物质, $2 \times$ 藻组 VFAs 含量可达 $2232.96 \mu \mathrm{l} / \mathrm{L}, 1 \times$ 藻组可达 $1263.36 \mu \mathrm{l} / \mathrm{L}$, 最高可达 $42.1 \%$, 为对照组 (无添加蓝藻) 的 2.43 倍, 从而促进了硝态氮和亚 硝态氮还原成 $\mathrm{N}_{2}$ 和 $\mathrm{N}_{2} \mathrm{O}$ 的过程, 提高氮素的去除率. 但底泥中沉降蓝澡需要一定的降解时间, 前 4 天添加冷冻干蓝藻粉 的处理组 COD 降解率较低, 电位处于正值, 体系中产生硝态氮, 随后 COD 持续降低, 添加 $2 \times$ 藻组 COD 最大去除率为 $42.08 \%, 1 \times$ 藻组为 $32.93 \%$, 对照组仅为 $14.46 \%$, 表明藻细胞中的碳素已开始被利用. 本研究表明沉降蓝藻细胞能够为 底泥中的反硝化过程提供可利用碳源, 并深人揭示了沉降蓝藻作为碳源促进底泥反硝化过程的机理和对底泥中 $\mathrm{C} 、 \mathrm{~N}$ 的 影响,为在湖泊治理中降低氮素的内源污染提供了新的科学依据.
\end{abstract}

关键词: 沉降蓝藻; 碳源; 反硝化; 脱氮; 挥发性脂肪酸; 太湖

\section{Effect and mechanism of algae bloom on the denitrification processes in the sediments of Lake Taihu}

\author{
LI Liwen ${ }^{1,2}$, PAN Gang ${ }^{2}$, LI Liang ${ }^{2}$, LI Hong ${ }^{2}$, SHI Wenqing ${ }^{2}$, ZHANG Honggang ${ }^{2} \&$ ZHU Guangwei ${ }^{1}$ \\ (1: Nanjing Institute of Geography and Limnology, Chinese Academy of Sciences, Nanjing 210008, P. R. China) \\ (2: Research Center for Eco-environmental Sciences, Chinese Academy of Sciences, Beijing 100085, P. R. China)
}

Abstract: Denitrification in anaerobic lake bottom sediments and leachate providing carbon source via flocculated algae were simulated in the laboratory. TN, ammonium, nitrite, nitrate, volatile fatty acids ( VFAs), COD, electric potential and pH were analyzed to confirm that the denitrification in sediments was truly promoted by small molecular compounds such as VFAs which were reduced from the plentiful biodegradable carbon in algae. The maximum VFAs content in $2 \times$ algae system and $1 \times$ algae system were 2232.96 and $1263.36 \mu \mathrm{l} / \mathrm{L}$, respectively. The maximum TN removal ratio was about $42.1 \%$, nearly 2.43 times of the control. The carbon of flocculated algae is available for heterotrophic microbes but needs some time to be transformed. A sustaining COD reduction rate showed in all the added algae systems after low COD reduction rate, positive electric potential and increasing nitrate had last in them for about 4 days. The maximum COD reduction rate of $2 \times$ algae system, $1 \times$ algae system and the control was $42.08 \%, 32.93 \%$ and $14.46 \%$, respectively. The degradation of algae generated VFAs can be directly used by denitrifying bacteria to facilitate denitrification process. The effect that flocculated algae cell as carbon source can promote sediment denitrifying process was verified, and the influence of flocculated algae on $\mathrm{C}$ and $\mathrm{N}$ of sediments was analyzed. The study could provide technical basis for reducing the internal nitrogen in sediments of eutrophic lakes.

Keywords: Flocculated algae; carbon source; denitrification; nitrogen removal; volatile fatty acids; Lake Taihu

湖泊水体中的化合态氮素大部分通过固氮生物和大气沉降而来, 这些化合态氮素又可以通过水生生物 的吸收利用以及微生物的硝化和反硝化作用等转化为生物量或者氮气从湖泊中去除, 从而维持湖泊中氮素

* 国家重点基础研究发展计划“973”项目(2010CB933600)资助. 2012-12-18 收稿;2013-02-04 收修改稿. 黎丽 雯,女,1988 年生, 硕士研究生;E-mail: liliwen_1988@ hotmail. com.

** 通信作者;E-mail: gapn@ rcees. ac. cn, gwzhu@ niglas. ac. cn. 
循环的平衡状态 ${ }^{[1]}$. 但是人类活动, 如大规模的合成氨工业以及大量施用化肥, 使得大量外源性氮素进人湖 泊,并在湖泊底泥中富集 ${ }^{[1-2]}$. 很多研究均已表明, 湖泊氮素的增加对于环境有持久的不良影响,包括污染饮 用水、导致水体富营养化等 ${ }^{[2]}$. 淡水的富营养化是全球关注的热点问题, 湖泊富营养化的典型标志之一就是 蓝藻的暴发. 藻华的暴发也伴随着蓝藻在湖体中的自然沉降. 在戻氧情况下,除了底泥营养盐的释放, 藻类 残体的分解也可以产生大量颗粒态、溶解态和胶体态的氮、有机碳 ${ }^{[3]}$. 已有研究表明,美国 Narragansett Bay 藻量与沉积物中反硝化过程存在相关关系 ${ }^{[4]}$. 但沉降的蓝藻促进反硝化作用的机制以及如何影响底泥中的 $\mathrm{C} 、 \mathrm{~N}$ 未见报道.

反硝化脱氮同物理、化学除氮法相比, 不是将硝酸盐简单地浓缩, 而是将它转化为 $\mathrm{N}_{2}$ 和 $\mathrm{N}_{2} \mathrm{O}$, 被认为是 湖泊中去除氮元素的最主要生物途径 ${ }^{[5]}$. 反硝化菌大部分为异养型厌氧微生物, 需要碳源提供能量以及电 子供体以实现氮素的降解 ${ }^{[8]}$. 生物脱氮过程中, 电子供体通常来源于可生物降解的溶解性有机物. 在过去的 几十年中, 大量的研究尝试补充各种碳源以促进反硝化的进行 ${ }^{[6-7]}$. 已有研究表明往太湖底泥中添加淀粉和 乙酸钠可促进底泥总氮 ( TN ) 的去除 ${ }^{[6]}$. 但是外加碳源可能会涉及巨大的经济投人和增加环境负担 ${ }^{[8]}$. 藻细 胞含有比农作物更高的脂类、可溶性多糖和蛋白质等, 已被用于制造生物柴油, 也被认为是生物可降解的有 机物 ${ }^{[9-10]}$. 沉降到底泥中的蓝藻若可以为底泥反硝化菌提供碳源, 对充分利用资源和降低成本来说都具有重 要意义,但是关于蓝藻提高底泥反硝化菌脱氮能力的可行性以及其效率的研究很少.

絮凝除藻技术已被认为是一种较好的从水体中去除藻类水华的方法. 潘纲等不断改进改性黏土技术， 提出改性当地土壤絮凝除藻技术 (MLS 技术). 该技术利用湖泊周围易得、无污染、廉价的土壤, 通过微生物 可降解的壳聚糖改性, 可将湖泊中 $88 \% \mathrm{TN}$ 和 $89 \%$ 的藻华去除 ${ }^{[1-12]}$. 通过絮凝除藻技术将藻体从水体中转移 至底泥表面, 可能会给底泥微生物提供大量的可生物降解有机碳并且提供电子供体, 从而影响微生物的生 物地球化学作用. 因此需要研究沉降藻体对底泥微生物活动的影响, 为絮凝技术在湖泊治理中降低氮素的 内源污染提供新的技术依据.

本文通过向太湖底泥中添加不同量的藻细胞以模拟蓝藻沉降后与底泥的混合状态, 监测底泥中 TN 、 $\mathrm{pH} 、 \mathrm{Eh} 、 \mathrm{COD}$ 、挥发性脂肪酸( VFAs) 和氮元素转化, 研究藻细胞的存在是否会对底泥中氮去除产生影响, 并 探究其影响机制, 为富营养化湖泊氮内源污染的治理提供新的科学依据.

\section{1 材料与方法}

\section{1 样品采集}

底泥和蓝藻样品均采集于太湖北部檀溪湾 $\left(31^{\circ} 26^{\prime} 42^{\prime \prime} \mathrm{N}, 120^{\circ} 07^{\prime} 49^{\prime \prime} \mathrm{E}\right.$ ), 用柱状采泥器 (有机玻璃柱, 内 径 $8.4 \mathrm{~cm}$, 高 $50 \mathrm{~cm}$ ) 于 2012 年 11 月 10 日采集 6 个表层 $10 \mathrm{~cm}$ 底泥样品, 置于冰盒中送至实验室, 混合均匀 后 $4{ }^{\circ} \mathrm{C}$ 下保存. 用浮游植物网收集水体表面蓝藻, 置于冰盒中送至实验室, 用 $0.5 \%$ 的 $\mathrm{NaCl}$ 溶液清洗 3 遍后 离心富集, 冷冻干燥后备用. 冷冻干燥是一种可以保证微生物保藏期长、成活率高的干燥方式 ${ }^{[13]}$. 采用此种 方式可以极大程度地保证细胞活性、藻体内碳素和氮素等成分, 将其作为实验材料尽可能地表达沉降蓝藻 在底泥中的变化以及 $\mathrm{C} 、 \mathrm{~N}$ 情况. 经元素分析仪 (Vario EL III) 测得底泥中 $\mathrm{N}$ 含量为 $0.110 \%, \mathrm{C}$ 含量为 $0.712 \%$; 藻粉中 $\mathrm{N}$ 含量为 $9.679 \%$, C 含量为 $45.807 \%$.

\section{2 厌氧培养实验}

为了屏蔽掉天然水体中溶解性碳素和其他物质对于实验的干扰作用, 以便更清唽地探究蓝藻作为底泥 反硝化菌碳源的效果和机理, 实验体系为将 $30 \mathrm{~g}$ 底泥 (干重) 和 $300 \mathrm{ml}$ 人工湖水 ( $1 \mathrm{mg} / \mathrm{L} \mathrm{NH}_{4} \mathrm{Cl}, 1 \mathrm{mg} / \mathrm{L}$ $\left.\mathrm{KNO}_{3}, 0.1 \mathrm{mg} / \mathrm{L} \mathrm{NaNO}_{2}\right)^{[6]}$ 置于 $500 \mathrm{ml}$ 灭菌三角瓶中. $1 \mathrm{~g}$ 藻粉和 $2 \mathrm{~g}$ 藻粉分别添加于上述三角瓶体系中构 成 $1 \times$ 藻组和 $2 \times$ 藻组; 无藻粉添加的上述三角瓶体系构成对照组. 因土壤部分理化性质易受到传统灭菌方 法如高压高温灭菌影响 ${ }^{[14]}$, 但采能抑制藻类光合作用, 影响叶绿素合成, 使藻细胞发生畸变并损伤细胞膜导 致细胞膜通透性增大 ${ }^{[15-16]}$, 对土壤微生物有杀灭作用而对土壤理化性质影响较小 ${ }^{[17]}$. 因此采用 $1 \mathrm{~g}$ 藻粉和 $0.4 \mathrm{~g} \mathrm{HgCl}_{2}$ 添加于上述三角瓶体系中构成灭菌对照组 ${ }^{[17]}$. 所有处理均做 3 次重复. 实验开始前及每次取样 后, 向所有处理中通人 $10 \mathrm{~min} \mathrm{~N}_{2}$ 以去除瓶中氧气, 然后橡胶塞密封, 置于振荡培养箱中 150 转 $/ \mathrm{min}, 25^{\circ} \mathrm{C}$ 培 养 $21 \mathrm{~d} . \mathrm{pH}$ 和 Eh 用 $\mathrm{pH}$ 电极测定 (Thermo Orion Model 250, USA). 定期采集 $10 \mathrm{ml}$ 泥水混合物置于 $10 \mathrm{ml}$ 离 心管中,用于 COD、TN、化合态氮素和挥发性脂肪酸(VFAs) 的测定. 


\section{3 样品化学分析}

泥水混合物中 TN 的测定采用碱性过硫酸钾消解法 ${ }^{[18]}$. COD 用哈希 COD 测定仪 (HACH DRB200, DB2800) 测定. 各实验组在泥水混合状态下的 TN、COD 浓度初始值为: 对照组 TN 浓度为 $15.33 \mathrm{mg} / \mathrm{L}, \mathrm{COD}$ 浓度为 $32.5 \mathrm{mg} / \mathrm{L}$; 灭菌对照组和 $1 \times$ 藻组 TN 浓度均为 $37.26 \mathrm{mg} / \mathrm{L}$, COD 浓度均为 $99.31 \mathrm{mg} / \mathrm{L} ; 2 \times$ 藻组 TN 浓度为 $49.35 \mathrm{mg} / \mathrm{L}, \mathrm{COD}$ 浓度为 $154.49 \mathrm{mg} / \mathrm{L}$. VFAs 采用比色测定法, 用不同浓度乙酸溶液做标准曲线 ${ }^{[19]}$. 剩余泥水混合物在 7000 转 $/ \min$ 下离心 $8 \mathrm{~min}$ 并且通过 $0.45 \mu \mathrm{m}$ 的针式过滤器用于化合态氮素和挥发性脂 肪酸的测定. 为了维持系统稳定性, 铵态氮 $\left(\mathrm{NH}_{4}^{+}-\mathrm{N}\right)$ 、硝态氮 $\left(\mathrm{NO}_{3}^{-}-\mathrm{N}\right)$ 、亚硝态氮 $\left(\mathrm{NO}_{2}^{-}-\mathrm{N}\right)$ 均参照微缩的光 度法用酶标仪 (Varioskan Flash) 测定, 铵态氮的测定采用水杨酸一次氯酸盐光度法, 硝态氮的测定采用镉还 原法,亚硝态的测定采用 $\mathrm{N}-$ - (1-荟基) -乙二胺光度法 ${ }^{[20-21]}$.

\section{2 结果与讨论}

\section{1 底泥 TN 去除率}

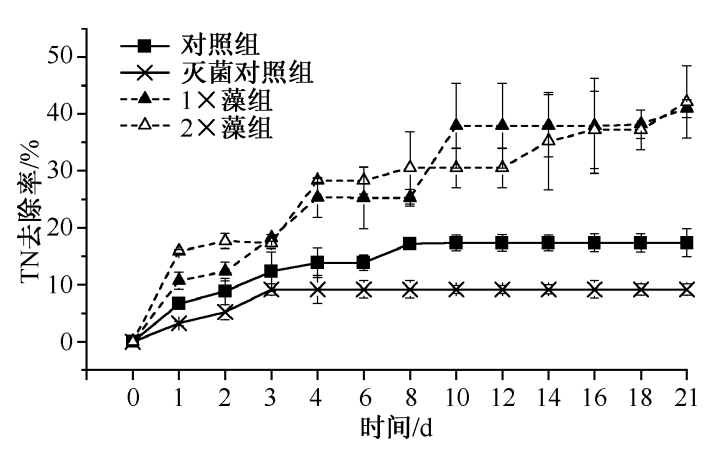

图 1 各实验处理组中底泥 TN 去除率

Fig. 1 TN removal ratio of sediments in each system

实验处于厌氧条件下, 底泥中 $\mathrm{TN}$ 的去除主要 依靠反硝化过程, 用底泥 TN 的去除率来表征藻增 强底泥反硝化的效果 ${ }^{[6]}$. 在 $21 \mathrm{~d}$ 的实验里, 各实验 处理组的 TN 去除率如图 1 所示.

灭菌对照组在前 $3 \mathrm{~d}$ 对 TN 有一定的去除效果 (最高为 $9.17 \%$ ), 这可能跟 $\mathrm{HgCl}_{2}$ 杀死微生物需要 一定时间有关. 添加藻的处理组 TN 去除率均比对 照组和灭菌对照组要高, 最高可达到 $42.1 \%$, 为对 照的 2.43 倍, 说明藻的添加促进了反硝化反应的进 行. 总体上, $1 \times$ 藻组和 $2 \times$ 藻组具有相同变化趋势, $1 \mathrm{~g}$ 和 $2 \mathrm{~g}$ 太湖蓝藻生物量作为底泥反硝化菌的碳源 以及促进反硝化脱氮效率上, 不存在显著差异 $(t$ 检 验, $P=0.95)$.

虽然蓝藻细胞也增加了底泥中氮素的含量, 但经过 $21 \mathrm{~d}$ 实验后, 各实验组样品中氮素含量均低于初始浓度, 灭菌对照组的 TN 浓度最高 $(33.84 \mathrm{mg} / \mathrm{L})$, 其次为 $2 \times$ 藻组 $(28.57 \mathrm{mg} / \mathrm{L})$, 再次是 $1 \times$ 藻组 $(22.00 \mathrm{mg} / \mathrm{L})$, 均 高于对照组 $(12.67 \mathrm{mg} / \mathrm{L})$. 通过计算实验体系中氮素去除量与剩余量的比值可得, $1 \times$ 藻组 $(69.36 \%)$ 和 $2 \times$ 藻组 $(72.73 \%$ ) 均大于对照组 (20.99\%) 和灭菌对照组 (10.11\%). 由此可知, 添加蓝藻的实验组显著提 高了底泥微生物的脱氮效率. 值得注意的是, 本实验中仅依靠蓝藻对底泥中土著反硝化菌脱氮的促进作用, 并不能完全去除由于蓝藻添加导致的氮素增量, 可能是由于本实验周期较短 (21 d), 而在实际天然湖泊中, 底泥微生物的反硝化作用是长时间的持续过程, 只要碳源充足, 由沉降蓝藻导致底泥中的碳素增量也可以 逐渐被去除.

\section{$2.2 \mathrm{COD}$ 去除率和 Eh 变化}

COD 去除率变化可以用来表征体系中有机物质的降解程度, 也间接表征体系中微生物利用碳素的情 况. Eh 表征氧化还原状况, 它与体系中氧化还原物质的含量、溶解氧浓度、酸性物质等有关, 与体系的氧化还 原反应呈正相关关系 ${ }^{[22]}$. 灭菌对照组中 COD 浓度几乎没有变化, 最大去除率仅为 $2.63 \%$, 对照组、2 $\times$ 藻组 和 $1 \times$ 藻组 COD 最大去除率分别为 $14.46 \% 、 42.08 \%$ 和 $32.93 \%$ (图 $2 \mathrm{~A}$ ), 说明藻体中高含量的碳素大部分 为可生物降解的物质, 并且在厌氧情况下得到有效分解. 前 $6 \mathrm{~d}$ 添加藻的处理组 COD 浓度变化不大, 甚至有 少量增加, 对照组 COD 浓度减小的速率大于添加藻的处理组, 这可能是因为藻体转化为反硝化菌可直接利 用的物质需要一定的降解时间. 第 $10 \mathrm{~d}$ 后 $1 \times$ 藻组和 $2 \times$ 藻组 COD 浓度持续降低并且低于对照组, 说明异 养微生物已经开始有效分解藻细胞.

实验结束后, $1 \times$ 藻组 $(66.61 \mathrm{mg} / \mathrm{L})$ 和 $2 \times$ 藻组 $(89.48 \mathrm{mg} / \mathrm{L})$ 的 COD 浓度均高于对照组 $(27.80 \mathrm{mg} / \mathrm{L})$. 计算实验体系中 COD 去除量与剩余量的比值可得, $1 \times$ 藻组 ( $48.63 \%$ ) 和 $2 \times$ 藻组 (72.71\%) 均大于对照组 ( $15.11 \%)$ 和灭菌对照组 $(2.38 \%)$. 由此可见, 在底泥微生物的作用下, 藻体中被有效降解的部分比底泥中 
有效降解的部分大许多, 说明蓝藻中可生物利用

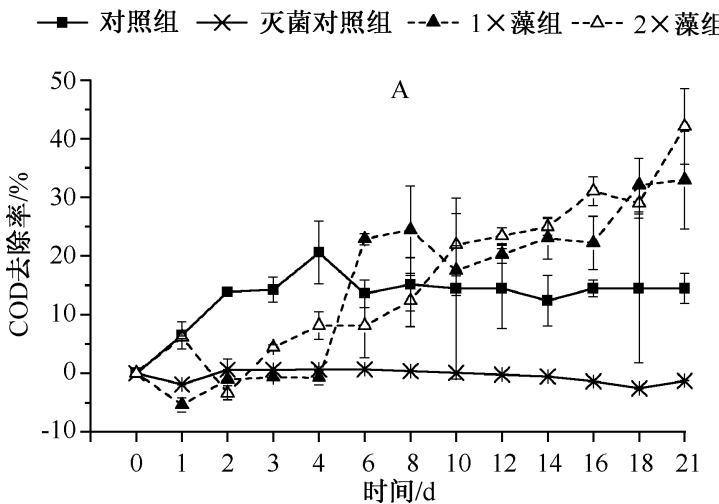
的部分高于底泥中的有机质. 根据文献可知, 蓝 藻中的蛋白质占藻体干重的 $30 \% \sim 70 \%$,脂肪占 $2 \% \sim 5 \%$, 产生于细胞外的多糖占 $16 \% \sim$ $41 \%{ }^{[23-24]}$, 可生物降解部分占藻体干重的 50\% $70 \%$,在最佳条件下可达 $90 \%$ 以上 ${ }^{[25]}$,但如何 提高其降解率, 更好地给底泥中反硝化菌提供 能量和电子供体, 以促进脱氮作用需要进一步 研究.

灭菌对照组中电位波动较小, 一直处于 $100 \mathrm{mV}$ 左右, 对照组中电位除了第 $1 \mathrm{~d}$ 、第 $14 \mathrm{~d}$ 为正值,其他时候处于 $-7.5 \sim-23.13 \mathrm{mV}$ 之间 (图 2B). $1 \times$ 藻组和 $2 \times$ 藻组电位有相同的变化 规律, 其中 $1 \times$ 藻组电位略低于 $2 \times$ 藻组. 当蓝藻 处于厌氧环境中,有的以发酵的形式,分解、利 用积累在细胞体内的糖原作为能量来源, 从而 可以维持其生命活动并生长良好, 而生长良好 的藻甚至可以产生氧气, 而不具备这种能力的 其他藻类在暴露于黑暗、厌氧条件下 $2 \sim 3 \mathrm{~h}$ 后, 细胞就会死亡和分解 ${ }^{[26]}$. 在第 $2 \sim 6 \mathrm{~d}, 1 \times$ 藻组 中电位为正值, 第 $2 \sim 14 \mathrm{~d}, 2 \times$ 藻组电位为正 值. 添加藻的处理组中实验初期的正电位可能 与部分藻在厌氧、黑暗培养条件下持续存活了 一定时间有关. 从第 $14 \mathrm{~d}$ 开始, 添加藻的处理组 中电位急剧下降, 表明还原反应如反硝化过程 占居主导地位, 藻体迅速降解成为了反硝化菌

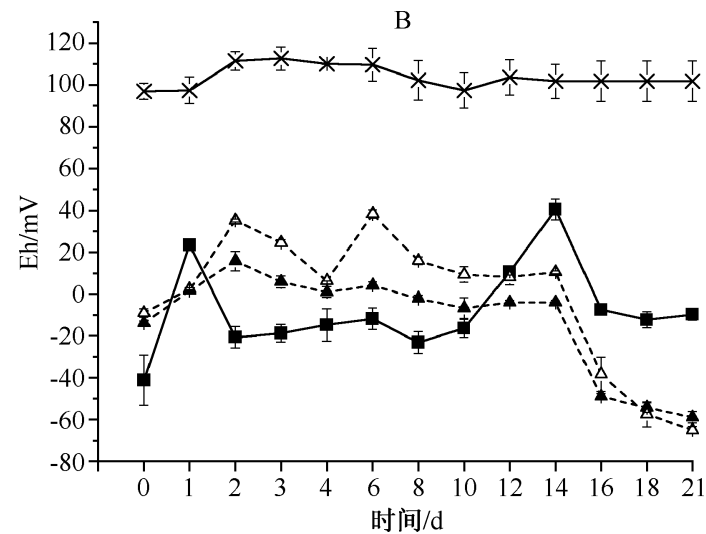

图 2 各实验处理组中 $\operatorname{COD}(\mathrm{A})$ 去除率和电位 $(\mathrm{B})$ 的变化

Fig. 2 The changes of COD removal ratio (A) and electric potential (B) in each system 的电子供体.

\section{3 挥发性脂肪酸的产生和 $\mathrm{pH}$ 变化}

在反硝化脱氮过程中,能够直接被反硝化菌利用的只有可溶解性、易生物降解的有机物,如乙酸、甲酸、丙酸等 低分子有机酸等,其他大分子有机物和不易生物降解的有机物必须先转化为低分子有机酸才能被微生物利 用 ${ }^{[7]}$. 甲酸、乙酸、丙酸、丁酸、戊酸等 10 个碳原子以下的脂肪酸统称为挥发性脂肪酸(VFAs). 在戻氧条件下, 通过厌氧发酵将蓝藻中有机物转化为挥发性脂肪酸 ${ }^{[27]}$, 从而给反硝化过程提供能量和电子供体. 对照组 VFAs 在初始阶段含量高, 随后逐渐减小, 而灭菌对照组与添加藻的处理组具有相同规律, VFAs 持续不断产 生, 总体上 VFAs 含量均高于对照组, 并且 $2 \times$ 藻组 VFAs 含量 (最高可达 $2232.96 \mu \mathrm{l} / \mathrm{L}$ ) 高于 $1 \times$ 藻组 (最高 可达 $1263.36 \mu \mathrm{l} / \mathrm{L}$ )，几乎与投加量呈正比关系(图 3A). 这表明添加藻可以为底泥中的反硝化菌提供大量 的 VFAs, 为其脱氮活动提供直接碳源, 且添加藻量与 VFAs 含量具有明显相关性. 实验开始时, 对照组的 VFAs 含量高于添加藻组和灭菌对照组, 这有可能与藻细胞的吸附作用有关 ${ }^{[28]}$, 而实验过程中添加藻组 (特 别是 $2 \times$ 藻组) 和灭菌对照组中 VFAs 含量的增加表明,这些体系中 VFAs 的排放通量大于吸收通量,但具体 原因还有待进一步研究. 在前 $3 \mathrm{~d}$, 灭菌对照组 VFAs 含量高于添加藻的处理组, 这说明 VFAs 的产生与藻细 胞核微生物的活性有关. 在灭菌对照组中因为 $\mathrm{HgCl}_{2}$ 的加人使得藻细胞在前 $3 \mathrm{~d}$ 迅速衰亡, 而在添加藻的处 理组中藻细胞是一个逐渐衰亡的过程. 灭菌对照组、 $1 \times$ 藻组、 $2 \times$ 藻组中 VFAs 含量的波动变化可能与通人 $\mathrm{N}_{2}$ 吹脱掉有关, 使得 VFAs 含量并未持续增加. 对照组未持续产生 VFAs, 故在 $\mathrm{N}_{2}$ 吹脱后, 呈现出 VFAs 浓度 下降的趋势. 

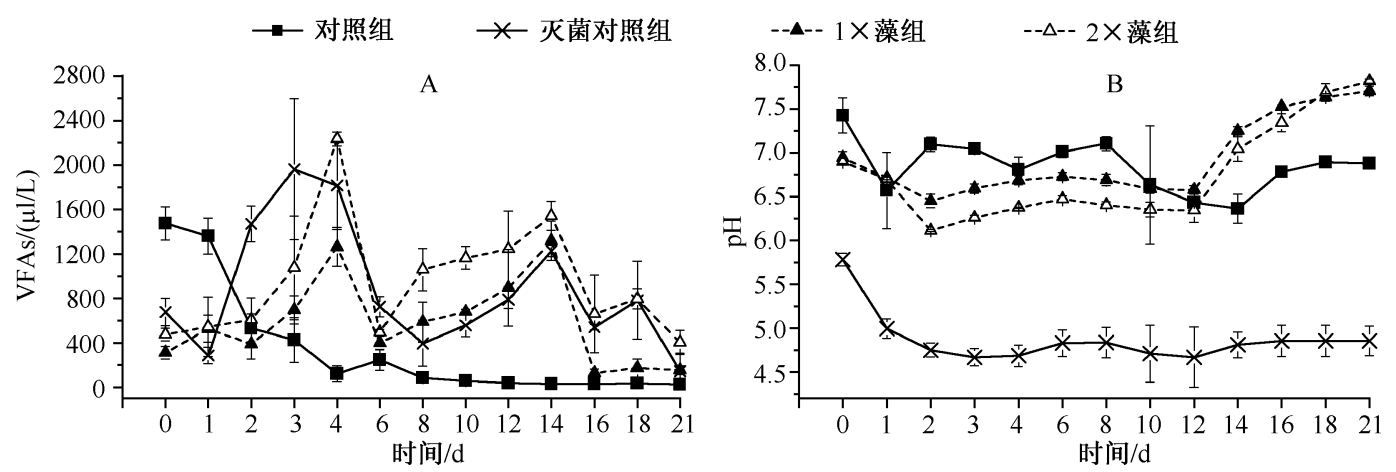

图 3 各实验处理组中 $\operatorname{VFAs}(\mathrm{A})$ 和 $\mathrm{pH}(\mathrm{B})$ 变化

Fig. 3 The changes of VFAs content(A) and $\mathrm{pH}$ (B) in each system

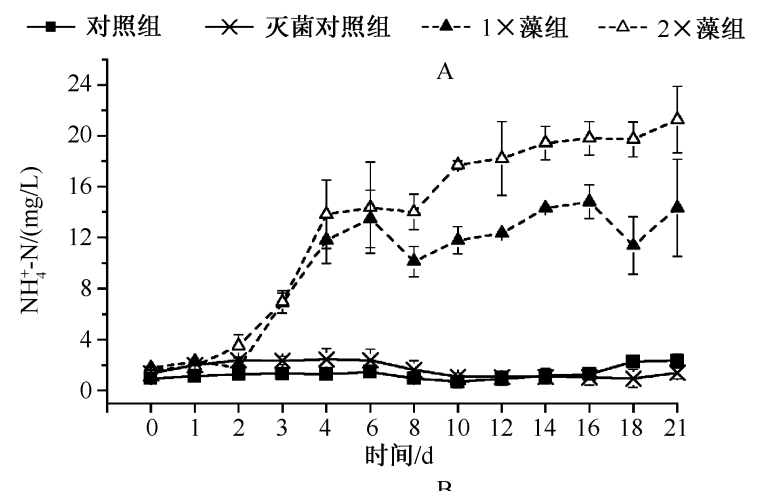

$\mathrm{pH}$ 是控制水一土界面污染物转化机制的重 要因素 ${ }^{[29]}$. 对照组中 $\mathrm{pH}$ 总体上处于 $6.36 \sim$ 7.42 之间; 灭菌对照组在前 $3 \mathrm{~d} \mathrm{pH}$ 从 5.78 持 续下降, 随后基本保持在 4.85 左右 (图 3B). 这 可能跟 $\mathrm{HgCl}_{2}$ 加人后产生大量的 VFAs, 并且微 生物失活使得体系中的 $\mathrm{pH}$ 缓冲体系遭到破坏 有关. 前 $12 \mathrm{~d}$, 添加藻的处理组 $\mathrm{pH}$ 处于对照组 和灭菌对照组之间, 并且 $2 \times$ 藻组的 $\mathrm{pH}$ 比 $1 \times$ 藻组的低, 这可能与藻的分解产生 VFAs 以及 底泥微生物对 $\mathrm{pH}$ 的缓冲作用有关. 随后, 添加 藻的处理组的 $\mathrm{pH}$ 发生逆转, 超过了对照组的 $\mathrm{pH}$, 并且在第 $18 \mathrm{~d}$ 和 $21 \mathrm{~d}$ 时 $2 \times$ 藻组的 $\mathrm{pH}$ 高 于 $1 \times$ 藻组的 $\mathrm{pH}$, 这可能与实验后期产生了大 量铵态氮等碱性物质有关.

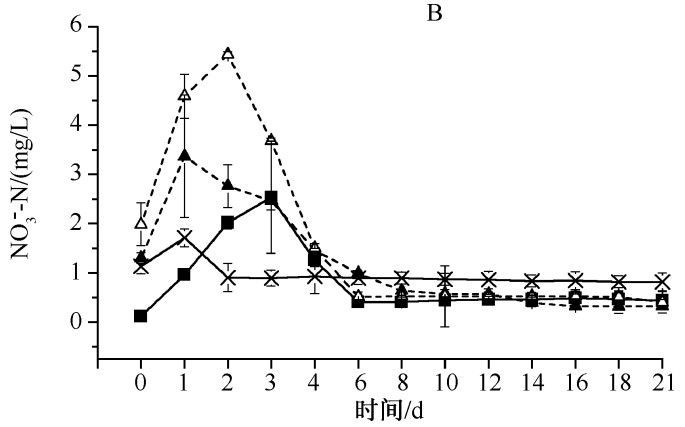

\section{4 化合态氮素的转化}

添加藻的处理组使得体系中的铵态氮浓度 比对照组高, 并且 $2 \times$ 藻组高于 $1 \times$ 藻组 (图 $4 \mathrm{~A}$ ). 铵态氮的持续增加同时也解释了第 $12 \mathrm{~d}$ 后 添加藻的处理组中 $\mathrm{pH}$ 变高的原因. $\mathrm{pH}$ 和电位 同样也影响着氮素的种类. 在第 $2 \sim 6 \mathrm{~d}, 1 \times$ 藻 组中电位为正值, 第 $2 \sim 14 \mathrm{~d}, 2 \times$ 藻组电位为正 值, 并且体系中 $\mathrm{pH}$ 保持在弱酸范围内 $(6.1 \sim$ $7.0)$, 此时底泥存在着较弱的氧化反应和还原 反应. 因此, 添加藻的处理组中 $\mathrm{NO}_{3}^{-}-\mathrm{N}$ 浓度先 增加再降低并且高于 2 个对照组, $1 \times$ 藻组最高 为 $3.37 \mathrm{mg} / \mathrm{L}, 2 \times$ 藻组最高为 $5.44 \mathrm{mg} / \mathrm{L}$ (图 $4 \mathrm{~B}) . \mathrm{NO}_{2}^{-}-\mathrm{N}$ 浓度在所有体系中均逐渐降低, 并 且添加藻的处理组下降幅度最高, 说明还原反 应进行得彻底 (图 4C). 实验结果也反映出氮素 在沉积物的迁移转化机制, 添加藻的处理组先 产生硝态氮, 在厌氧和碳源充足的情况下, 反硝 化菌再将硝态氮和亚硝态氮降解为气态氮. 


\section{5 环境意义}

藻华暴发的湖泊中必然伴随着藻类的自然沉降, 絮凝除藻技术在富营养化湖泊治理中的发展和应用更 是加大了湖泊底泥中藻细胞的沉积. 大量的沉积藻细胞经过衰亡、腐烂和降解等过程释放其体内的营养物 质,有可能会导致湖泊底泥中氮素和有机物的增加, 长期积累后可能变成湖泊富营养化的内源污染来源之 一. 而藻细胞由于含有很高的脂类、多糖和蛋白质等物质已被广泛研究并被用来提炼生物柴油等可利用生 物资源. 因此,如果能够将沉积在底泥中的大量藻细胞进行资源化利用, 不但能够降低湖泊内源污染的风 险, 而且还能够为湖泊水生态系统提供能量来源. 本研究通过模拟实验, 探究了厌氧情况下太湖蓝藻沉积对 底泥反硝化过程的影响及其机制, 研究证明沉积在底泥中的蓝藻细胞不但能够为底泥微生物的反硝化过程 提供可利用碳源, 而且还能提高底泥脱氮的效率, 同时不同的蓝藻沉积量对底泥的物理化学性质产生一定 影响. 本研究结果可以为富营养化湖泊尤其是暴发蓝藻水华的湖泊内源氮素污染的治理提供新的思路和理 论依据. 同时, 本研究还发现沉降蓝藻需要一定时间的降解过程,这也为絮凝除藻技术的完善提供了思路. 如潘纲等 ${ }^{[12]}$ 提出, 絮凝除藻技术实施后, 为了避免营养物质从沉降蓝藻和底泥中释放到水体, 需要适当的覆 盖技术对藻体以及营养盐进行封存, 再结合水草吸收或调节底泥中微生物群落结构来强化硝化一反硝化的 共同作用等, 最终提高湖泊底泥的脱氮率. 但如何提高蓝藻对底泥脱氮的促进作用还需要进一步进行实验 室以及野外原位实验研究.

\section{3 结论}

1) 沉降藻体的降解能够产生大量的挥发性脂肪酸,而挥发性脂肪酸可以直接被反硝化菌利用,使得产 生的硝态氮和亚硝态氮得以在反硝化菌的作用下还原成 $\mathrm{N}_{2}$ 和 $\mathrm{N}_{2} \mathrm{O}$, 从而提高 $\mathrm{TN}$ 去除率,最高可达 $42.1 \%$, 为对照组 (无添加蓝藻) 的 2.43 倍.

2) 沉降蓝藻的生物量影响底泥物理化学性质以及底泥反硝化过程,并且在戻氧、黑暗条件下藻体降解 需要一定时间,受到氧环境、微生物作用强度和降解时间的限制.

3) 对底泥土著反硝化菌的促进作用不足以全部去除蓝藻自身给沉积物带来的氮负荷,沉降的蓝藻可能 会使得底泥铵态氮、COD 浓度增加.

\section{4 参考文献}

[ 1 ] Canfield DE, Glazer AN, Falkowski PG. The evolution and future of Earth's nitrogen cycle. Science, 2010,330 (6001): 192-196.

[ 2 ] Galloway JN, Townsend AR, Erisman JW et al. Transformation of the nitrogen cycle: recent trends, questions, and potential solutions. Science, $2008, \mathbf{3 2 0}(5878)$ : 889-892.

[ 3 ] 孙小静,秦伯强,朱广伟. 蓝藻死亡分解过程中胶体态磷、氮、有机碳的释放. 中国环境科学, 2007,(3):341-345.

[ 4 ] Fulweiler RW, Nixon SW, Buckley BA et al. Reversal of the net dinitrogen gas flux in coastal marine sediments. Nature, $2007, \mathbf{4 4 8}(7150): 180-182$.

[ 5 ] Richardson WB, Strauss EA, Bartsch LA et al. Denitrification in the upper Mississippi River: rates, controls, and contribution to nitrate flux. Canadian Journal of Fisheries and Aquatic Sciences, 2004,61(7) : 1102-1112.

[ 6 ] Wang D, Huang Q, Wang C et al. The effects of different electron donors on anaerobic nitrogen transformations and denitrification processes in Lake Taihu sediments. Hydrobiologia, 2007,581 :71-77.

[ 7 ] 蔡碧婧. 反硝化脱氮补充碳源选择与研究 [学位论文].上海:同济大学,2008.

[8] 丁 怡,宋新山,严登华. 补充碳源提取液对人工湿地脱氮作用的影响. 环境科学学报,2012,(7):1646-1652.

[ 9 ] Banerjee C, Gupta P, Mishra S et al. Study of polyacrylamide grafted starch based algal flocculation towards applications in algal biomass harvesting. International Journal of Biological Macromolecules, 2012 ,51 (4) : 456-461.

[10] McGinn PJ, Dickinson KE, Bhatti S et al. Integration of microalgae cultivation with industrial waste remediation for biofuel and bioenergy production: opportunities and limitations. Photosynthesis Research, $2011, \mathbf{1 0 9}(1 / 2 / 3)$ : 231-247.

[11] Pan G, Yang B, Wang D et al. In-lake algal bloom removal and submerged vegetation restoration using modified local soils. Ecological Engineering, $2011,37(2)$ :302-308.

[12] Pan G, Dai L, Li L et al. Reducing the recruitment of sedimented algae and nutrient release into the overlying water using 
modified soil/sand flocculation-capping in eutrophic lakes. Environmental Science and Technology, 2012, 46 (9): 5077-5084.

[13] 李 华,骆艳娥,刘延琳. 真空冷冻干燥微生物的研究进展. 微生物学通报,2002,(3):78-82.

[14] 张 辉,张佳宝,赵炳梓等. 高温高压间歇灭菌对中国典型土壤性质的影响. 土壤学报,2011, (3):540-548.

[15] 崔建升, 齐彩亚, 刘小静. 铜绿微囊藻荧光对 $\mathrm{HgCl}_{2}$ 生物毒性响应规律的研究. 安徽农业科学, 2012, (24): 12148-12150.

[16] 牟 文, 熊 丽, 胡芹芹等. $\mathrm{HgCl}_{2}$ 对斜生栅藻 (Scenedesmus obliquus) 生理生化特性的影响. 生态毒理学报, 2009, (6) :854-859.

[17］李 梅,侯彦林,皮广洁. 施肥及种植作物对录污染土壤中微生物生态的修复. 生态环境,2004, (4):560-564.

[18］国家环境保护总局《水和废水监测分析方法》编委会. 水和废水监测分析方法:第 4 版. 北京: 中国环境科学出版 社,2002.

[19］任南琪,王爱杰. 厌氧生物技术原理与应用. 北京: 化学工业出版社,2004:315.

[20] Laskov C, Herzog C, Lewandowski J et al. Miniaturised photometrical methods for the rapid analysis of phosphate, ammonium, ferrous iron and sulfate in pore water of freshwater sediments. Limnology and Oceanography: Methods, 2007,5 :6371.

[21] Tu X, Xiao B, Xiong J et al. A simple miniaturised photometrical method for rapid determination of nitrate and nitrite in freshwater. Talanta, 2010,82(3) :976-983.

[22] Stumm W. Aquatic chemistry: An introduction emphasizing chemical equilibria in natural waters by Werner Strumm and James J. Morgan. New York: Wiley-Interscience, 1970.

[23] Menetrez MY. An overview of algae biofuel production and potential environmental impact. Environmental Science and Technology, 2012,46(13): 7073-7085.

[24] 丁艳华. 蓝藻综合利用的研究进展. 江苏环境科技,2008,(S1 ) :147-149.

[25] Becker W. Handbook of microalgal culture. Richmond: Blackwell, 2004:312-351.

[26] Stal LJ, Moezelaar R. Fermentation in cyanobacterial. FEMS Microbiology Reviews, 1997,21(2) :179-211.

[27] 李玉祥,刘 和,堵国成等. 碱性条件促进太湖蓝藻厌氧发酵产挥发性脂肪酸. 环境工程学报,2010,(1):209-213.

[28］逯亚峰. 滇池北岸蓝藻富集区水气界面挥发性有机物变化特征 [学位论文]. 昆明:云南大学,2010.

[29] Cao X, Chen Y, Wang X et al. Effects of redox potential and pH value on the release of rare earth elements from soil. Chemosphere, $2001, \mathbf{4 4}(4)$ : 655-661. 